\title{
Magnetic monopoles and dyons revisited: A useful contribution to the study of classical mechanics
}

\author{
Renato P dos Santos \\ PPGECIM, ULBRA - Lutheran University of Brazil, Av. Farroupilha, 8001 - Pr. 14, \\ S. 338 - 92425-900 Canoas, RS, Brazil \\ E-mail: renatopsantos@ulbra.edu.br
}

\begin{abstract}
Graduate level physics curricula in many countries around the world, as well as senior-level undergraduate ones in some major institutions, include Classical Mechanics courses, mostly based on Goldstein's textbook masterpiece. During the discussion of central force motion, however, the Kepler problem is virtually the only serious application presented. In this paper, we present another problem that is also soluble, namely the interaction of Schwinger's dual-charged (dyon) particles. While the electromagnetic interaction of magnetic monopoles and electric charges was studied in detail some 40 years ago, we consider that a pedagogical discussion of it from an essentially classical mechanics point of view is a useful contribution for students. Following a path that generalizes Kepler's problem and Rutherford scattering, we show that they exhibit remarkable properties such as stable non-planar orbits, as well as rainbow and glory scattering, which are not present in the ordinary scattering of two singly charged particles. Moreover, it can be extended further to the relativistic case and to a semi-classical quantization, which can also be included in the class discussion.
\end{abstract}

PACS numbers: 0140gb, 4520Jj, 0350De, 1480Hv

Keywords: Physics teaching, Classical Mechanics, Lagrangian mechanics, Electromagnetism, Dirac magnetic monopoles, dyons

Submitted to: Eur. J. Phys. 


\section{Introduction}

In Classical Mechanics courses, the so-called Kepler problem [1, ch. 3, sec. 3.7] is virtually the only serious completely integrable application of central forces discussed besides the harmonic oscillator. As Sivardière already pointed out in this journal [2], the motion of a charged particle in the field of a magnetic monopole [3], which is another example of completely integrable problem, is, unfortunately, not discussed.

Here, we extend and deepen Sivardière's study to a more general case, namely the interaction between Schwinger's dyons (dual charged particles) 4]. As it has formal similarity with the Kepler problem and the Rutherford scattering, we believe that it may be presented right after these ones in Classical Mechanics courses. Furthermore, it exhibits unusual features such as non-planar stable orbits and rainbow and glory scattering, results that may arouse students' and teachers' interest.

\section{A brief historical review of magnetic monopoles and dyons}

The similarity between the electric and magnetic fields is visible in Maxwell's equations. When deducing them in 1873, Maxwell himself pointed out that it would be necessary to assert that there are no net magnetic charged bodies and no 'magnetic currents' [5, art. 380, p. 6].

However, this symmetry would be restored if we were to assume the existence of a magnetic field density $\rho_{\mathrm{m}}$ and a 'magnetic current' $\boldsymbol{j}_{\mathrm{m}}$, obtaining (in SI units and with magnetic charges measured in ampere $\cdot$ meters)

$$
\begin{cases}\nabla \cdot \boldsymbol{E} & =\rho_{\mathrm{e}} / \epsilon_{0} \\ \nabla \times \boldsymbol{E} & =-\mu_{0} \boldsymbol{j}_{\mathrm{m}}-\partial \boldsymbol{B} / \partial t \\ \nabla \cdot \boldsymbol{B} & =\mu_{0} \rho_{\mathrm{m}} \\ \boldsymbol{\nabla} \times \boldsymbol{B} & =\mu_{0} \boldsymbol{j}_{\mathrm{e}}+\mu_{0} \epsilon_{0} \partial \boldsymbol{E} / \partial t\end{cases}
$$

In 1896, H. Poincaré, applied the concept of magnetic matter to explain Birkeland's magnetic deflection of cathode rays experiment. In it, he considered that the beam passes so close to one pole of the magnet that the other can be neglected [6]. This can be interpreted as a magnetic monopole approximation.

Nevertheless, the idea of a magnetic monopole as a particle having a single magnetic pole was introduced only in 1931 by Dirac, in his famous work [3].

As a matter of fact, Dirac was not looking for something like the monopole, but investigating the why of the quantization of electric charge. In other words, why the electric charge always appears in Nature as a multiple of the electron charge $e$ and why this charge has a value such that (in the same units as in (1))

$$
2 \epsilon_{0} h c / e^{2} \cong 137 \text {. }
$$

In that work, however, instead of the relation (2), he obtained

$$
e g / \epsilon_{0} h c^{2}=n \quad(n= \pm 1, \pm 2, \pm 3, \ldots),
$$


which is known as the Dirac quantization condition. It does not set a value for $e$, but only for its product by the magnetic charge $g$ of a hypothetical particle in its vicinity. On the other hand, Caruso [7] arrived at the same result (3) through a semi-classical derivation that gives it a new interpretation.

Despite that, in a way, (3) offers a solution to Dirac's initial problem: if there were a single magnetic monopole in the entire Universe, then all electric charges would be quantized according to (3). Therefore, in view of the observed quantization of electric charge and in the absence of another explanation for this fact at the time, the condition (3) was considered a serious argument for the existence of at least one magnetic monopole in the Universe.

Generalizing Dirac quantization condition, Schwinger [4], in 1969, introduced the dyons, which were suggested at the time as candidates for the quark model. Pinfold et al. 8] discuss the tremendous implications that the discovery of magnetic monopoles or dyons would have for our fundamental understanding of Nature at the deepest levels and describe the search for these particles, from Dirac's proposal in 1931 to the current MoEDAL experiment at CERN's LHC.

After this short historical account, we proceed to study the interaction of two dyons from a classical mechanics point of view.

\section{The classical mechanics of two dyons}

To formulate the Lagrangian for the electromagnetic interaction of two dyons, we need to circumvent the problem of what became known as the Dirac string [3]. It is a singularity line in the space starting from the monopole, over which the magnetic vector potential $\boldsymbol{A}$ does not satisfy the condition that the wave function is a univalent function.

Later on, Schwinger [9], Yang [10], and Wu and Yang [11] considered that the Dirac string has no physical meaning or real existence, being only an effect of the coordinate system, analogous to the problem of terrestrial geographic poles when trying to map the Earth's surface with a single chart. These authors stated that this problem can be solved by dividing the space around the monopole in two regions $a$ and $b$ and defining two potential vectors $\boldsymbol{A}_{a}$ and $\boldsymbol{A}_{b}$ which describe the monopole field in each of these regions and have singularities in the other $b$ and $a$ ones, respectively.

Bollini and Giambiagi [12], in their turn, proposed a multivalued distribution (generalized function) potential instead of a singular function. This approach, however, demands the use of the mathematical theory of distributions to the evaluation of its curls and divergences.

On the other hand, Sokolov [13] showed that the singularity of the magnetic monopole potential is of a purely kinematic origin, caused by the uncertainty of the azimuthal $\phi$ angle along the $z$ axis. As a consequence, for a charge in the field of a 'Coulombian' magnetic monopole

$$
\boldsymbol{B}=\frac{\mu_{0}}{4 \pi} \frac{g}{r^{3}} \boldsymbol{r}
$$


we can obtain the equation of motion, by using the Lorentz force

$$
\boldsymbol{F}=e \boldsymbol{v} \times \boldsymbol{B}
$$

and the expression (4) for the magnetic field, as

$$
\mu \frac{\mathrm{d} \boldsymbol{v}}{\mathrm{d} t}=\frac{\mu_{0}}{4 \pi} e g \boldsymbol{v} \times \frac{\boldsymbol{r}}{r^{3}}
$$

without the appearance of strings or fictitious fields as long as vector potentials and curls are written and evaluated in spherical coordinates in

$$
\mu \frac{\mathrm{d} \boldsymbol{v}}{\mathrm{d} t}=e \boldsymbol{v} \times(\boldsymbol{\nabla} \times \boldsymbol{A})_{\text {spherical }} .
$$

Sokolov's procedure can be as well generalized to the dyon-dyon case [14, p. 13] for any vector potential $\boldsymbol{A}$ whose curl evaluated in spherical coordinates furnishes the correct magnetic potential (4) such that (7) leads to (6). As a result, it is possible to obtain a classical string-free Lagrangian for the dyon-dyon case.

That being said, we will here build the dyon-dyon Lagrangian through a different procedure, by means of the fields instead of a potential. This procedure will lead, however, to a Lagrangian that matches Sokolov's one.

We start by noticing that Maxwell's equations (1) are invariant under the duality transformation $\boldsymbol{E} \rightarrow c \boldsymbol{B}, c \boldsymbol{B} \rightarrow-\boldsymbol{E}, \rho_{\mathrm{e}} \rightarrow \rho_{\mathrm{m}} / c$, and $\boldsymbol{j}_{\mathrm{e}} \rightarrow \boldsymbol{j}_{\mathrm{m}} / c$. That allows us to generalize Lorentz force to the dyon-dyon interaction and write the equation of motion as

$$
\begin{aligned}
\mu \frac{\mathrm{d} \boldsymbol{v}}{\mathrm{d} t} & =e_{1}(\boldsymbol{E}+\boldsymbol{v} \times \boldsymbol{B})+g_{1}\left(\boldsymbol{B}-\frac{1}{c^{2}} \boldsymbol{v} \times \boldsymbol{E}\right) \\
& =\left(e_{1} \frac{1}{4 \pi \epsilon_{0}} e_{2}+g_{1} \frac{\mu_{0}}{4 \pi} g_{2}\right) \frac{\boldsymbol{r}}{r^{3}}+\left(e_{1} \frac{\mu_{0}}{4 \pi} g_{2}-g_{1} \frac{1}{c^{2}} \frac{1}{4 \pi \epsilon_{0}} e_{2}\right) \boldsymbol{v} \times \frac{\boldsymbol{r}}{r^{3}} \\
& =\frac{1}{4 \pi \epsilon_{0}}\left(e_{1} e_{2}+\frac{1}{c^{2}} g_{1} g_{2}\right) \frac{\boldsymbol{r}}{r^{3}}+\frac{\mu_{0}}{4 \pi}\left(e_{1} g_{2}-g_{1} e_{2}\right) \boldsymbol{v} \times \frac{\boldsymbol{r}}{r^{3}} \\
& =\frac{1}{4 \pi \epsilon_{0}} q \frac{\boldsymbol{r}}{r^{3}}-\frac{\mu_{0}}{4 \pi} \kappa \boldsymbol{v} \times \frac{\boldsymbol{r}}{r^{3}}
\end{aligned}
$$

where

$$
\begin{aligned}
& q=e_{1} e_{2}+g_{1} g_{2} / c^{2} \\
& \kappa=e_{1} g_{2}-g_{1} e_{2},
\end{aligned}
$$

being $e_{1}, e_{2}, g_{1}$, and $g_{2}$ the electric and magnetic charges of the two dyons, corresponding the index ${ }_{2}$ to the dyon that remains at the origin of the relative coordinate system, $c$ the speed of light in a vacuum, and $\mu$, naturally, the system reduced mass, given by

$$
\mu=\frac{m_{1} m_{2}}{m_{1}+m_{2}} .
$$

To evaluate the cross product in (8), we need to express $\boldsymbol{r}$ and $\boldsymbol{v}$ in spherical coordinates as (see Figure 1)

$$
\begin{aligned}
& \boldsymbol{r}=r \hat{\boldsymbol{r}} \\
& \boldsymbol{v}=\dot{r} \hat{\boldsymbol{r}}+r \dot{\theta} \hat{\boldsymbol{\theta}}+r \sin \theta \dot{\phi} \hat{\boldsymbol{\phi}} .
\end{aligned}
$$


Now, we can write the force on the right-hand side of (8) as

$$
\begin{aligned}
\boldsymbol{F} & =\frac{1}{4 \pi \epsilon_{0}} \frac{q}{r^{2}} \hat{\boldsymbol{r}}-\frac{\mu_{0}}{4 \pi}\left(\frac{\kappa}{r} \sin \theta \dot{\phi} \hat{\boldsymbol{\theta}}+\frac{\kappa}{r} \dot{\theta} \hat{\boldsymbol{\phi}}\right) \\
& =\frac{1}{4 \pi \epsilon_{0}} \frac{q}{r^{2}} \hat{\boldsymbol{r}}+\mathscr{F},
\end{aligned}
$$

where $\mathscr{F}$ includes the non-central terms of $\boldsymbol{F}$ (those that not depend only on the distance $r$ and are not directed along the $\hat{\boldsymbol{r}}$ direction).

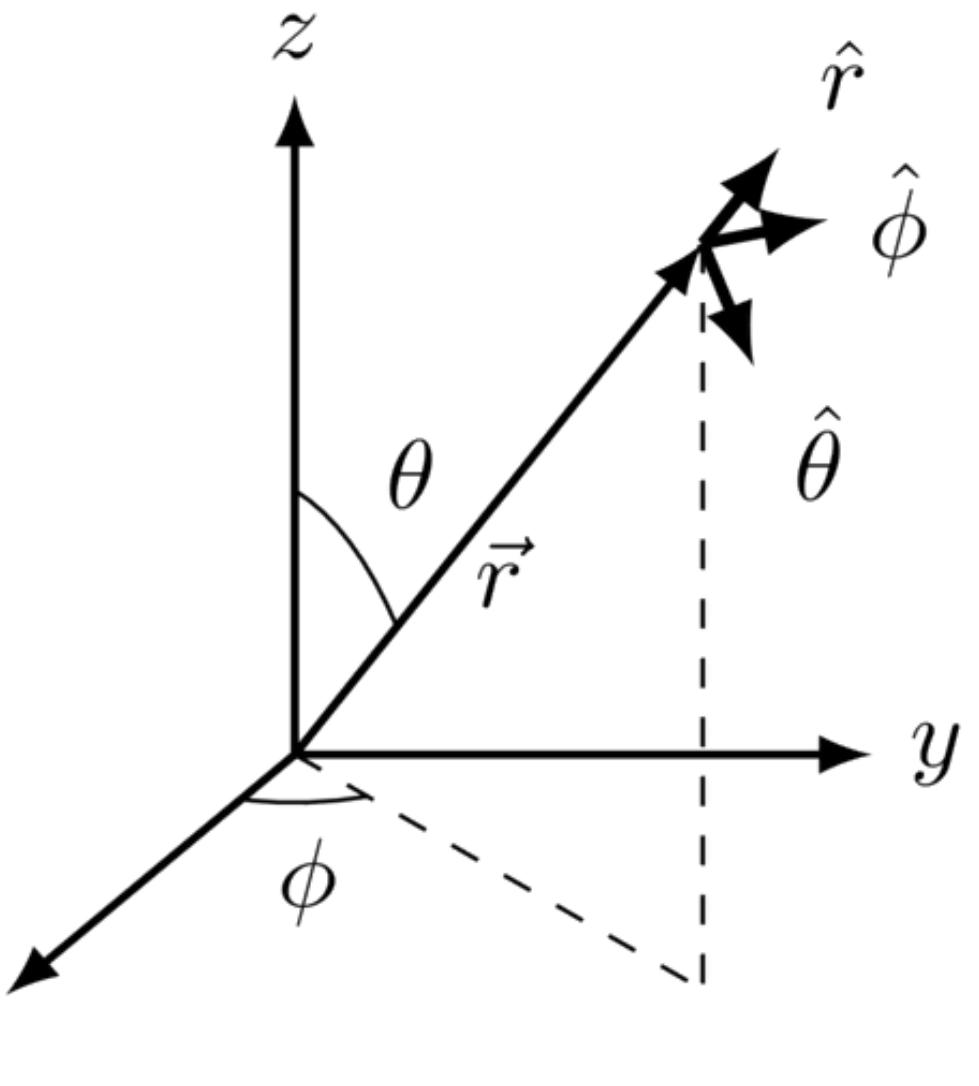

Figure 1. Position vector $(\boldsymbol{r})$ in the spherical coordinate system used in this paper.

From (11) and (12), we can write the Lagrangian

$$
\begin{aligned}
\mathscr{L} & =T-V \\
& =\frac{1}{2} \mu \dot{r}^{2}+\frac{1}{2} \mu r^{2} \dot{\theta}^{2}+\frac{1}{2} \mu r^{2} \sin ^{2} \theta \dot{\phi}^{2}-\frac{1}{4 \pi \epsilon_{0}} \frac{q}{r},
\end{aligned}
$$

which we call 'incomplete' because it contains only the term in 12 that is derivable from a scalar (Coulombian) potential.

As not all the forces acting on the system are derivable from a scalar potential, then Lagrange's equations can be written in the inhomogeneous form [1, sec. 1.5]

$$
\frac{\mathrm{d}}{\mathrm{d} t}\left(\frac{\partial \mathscr{L}}{\partial \dot{q}_{i}}\right)-\frac{\partial \mathscr{L}}{\partial q_{i}}=Q_{i} \quad(i=1,2,3, \cdots),
$$


where $Q_{i}$ are the generalized forces defined by

$$
Q_{i}=\mathscr{F}_{i} \cdot \frac{\partial \boldsymbol{r}}{\partial q_{i}}
$$

Substituting (13), (12) and (15) into (14), we obtain the equations of motion as

$$
\begin{aligned}
& \mu \ddot{r}-\mu r \dot{\theta}^{2}-\mu r \sin ^{2} \theta \dot{\phi}^{2}-\frac{1}{4 \pi \epsilon_{0}} \frac{q}{r^{2}}=0 \\
& \mu r^{2} \ddot{\theta}+2 \mu r \dot{r} \dot{\theta}-\mu r^{2} \sin \theta \cos \theta \dot{\phi}^{2}=-\frac{\mu_{0}}{4 \pi} \kappa \sin \theta \dot{\phi} \\
& \mu r^{2} \sin ^{2} \theta \ddot{\phi}+2 \mu r^{2} \sin \theta \cos \theta \dot{\theta} \dot{\phi}+2 \mu r \dot{r} \sin ^{2} \theta \dot{\phi}^{2}=\frac{\mu_{0}}{4 \pi} \kappa \sin \theta \dot{\theta} .
\end{aligned}
$$

It is worthy of note, however, that the equations of motion (16) could also have been obtained from a Lagrangian of the form

$$
\mathscr{L}=\frac{1}{2} \mu \dot{r}^{2}+\frac{1}{2} \mu r^{2} \dot{\theta}^{2}+\frac{1}{2} \mu r^{2} \sin ^{2} \theta \dot{\phi}^{2}-\frac{1}{4 \pi \epsilon_{0}} \frac{q}{r}+\frac{\mu_{0}}{4 \pi} \kappa \cos \theta \dot{\phi}
$$

which we call the 'minimal' Lagrangian as it is the simpler one that furnishes the equations (16) that describe the classical interaction of two dyons without the appearance of Dirac strings, according to our generalization of Sokolov's procedure.

Now, it is important to note that, since the interaction force 12 is not central, we should not expect the vector mechanical angular momentum $\boldsymbol{L}$ to be conserved. As a matter of fact, from its expression in spherical coordinates, using (11) again,

$$
\begin{aligned}
\boldsymbol{L} & =\mu \boldsymbol{r} \times \boldsymbol{v} \\
& =\mu r^{2} \sin \theta \dot{\phi} \hat{\boldsymbol{\theta}}+\mu r^{2} \dot{\theta} \hat{\boldsymbol{\phi}},
\end{aligned}
$$

we can evaluate its temporal derivative by remembering that

$$
\begin{aligned}
& \frac{\mathrm{d} \hat{\boldsymbol{r}}}{\mathrm{d} t}=\dot{\theta} \hat{\boldsymbol{\theta}}+\sin \theta \dot{\phi} \hat{\boldsymbol{\phi}} \\
& \frac{\mathrm{d} \hat{\boldsymbol{\theta}}}{\mathrm{d} t}=-\dot{\theta} \hat{\boldsymbol{r}}+\cos \theta \dot{\phi} \hat{\boldsymbol{\phi}} \\
& \frac{\mathrm{d} \hat{\boldsymbol{\phi}}}{\mathrm{d} t}=-\sin \theta \dot{\phi} \hat{\boldsymbol{r}}-\cos \theta \dot{\phi} \hat{\boldsymbol{\phi}},
\end{aligned}
$$

obtaining traightforwardly

$$
\begin{aligned}
& \left(\frac{\mathrm{d} \boldsymbol{L}}{\mathrm{d} t}\right)_{r}=0, \\
& \left(\frac{\mathrm{d} \boldsymbol{L}}{\mathrm{d} t}\right)_{\theta}=-\mu r^{2} \sin \theta \ddot{\phi}-2 \mu r^{2} \cos \theta \dot{\theta} \dot{\phi}-2 \mu r \dot{r} \sin \theta \dot{\phi}^{2} \quad, \text { and } \\
& \left(\frac{\mathrm{d} \boldsymbol{L}}{\mathrm{d} t}\right)_{\phi}=\mu r^{2} \ddot{\theta}+2 \mu r \dot{r} \dot{\theta}-\mu r^{2} \sin \theta \cos \theta \dot{\phi}^{2}
\end{aligned}
$$

Comparing these results with the left-hand sides of the two last equations of motion (16) above, we conclude that

$$
\begin{aligned}
& \left(\frac{\mathrm{d} \boldsymbol{L}}{\mathrm{d} t}\right)_{\theta}=-\frac{\mu_{0}}{4 \pi} \kappa \dot{\theta} \text { and } \\
& \left(\frac{\mathrm{d} \boldsymbol{L}}{\mathrm{d} t}\right)_{\phi}=-\frac{\mu_{0}}{4 \pi} \kappa \sin \theta \dot{\phi}
\end{aligned}
$$


or

$$
\frac{\mathrm{d} \boldsymbol{L}}{\mathrm{d} t}=-\frac{\mu_{0}}{4 \pi} \kappa \frac{\mathrm{d} \hat{\boldsymbol{r}}}{\mathrm{d} t}
$$

and that the vector

$$
\boldsymbol{J} \equiv \boldsymbol{L}+\frac{\mu_{0}}{4 \pi} \kappa \hat{\boldsymbol{r}}
$$

is conserved.

The vector $\boldsymbol{J}$, defined by (22), is known as the Poincaré integral of motion, as it was first found by Poincaré in his previously mentioned work [6]. It can be interpreted as the 'total' angular momentum of the system because the second term in the right-hand side of 22 is the angular momentum of the electromagnetic field, as demonstrated by Thomson [15, p. 532].

On the other hand, from the definition 22 and the fact that $\boldsymbol{L}$ is perpendicular to $\hat{\boldsymbol{r}}$, we also obtain

$$
J^{2}=L^{2}+\left(\frac{\mu_{0}}{4 \pi}\right)^{2} \kappa^{2}
$$

and, since $\boldsymbol{J}$ is conserved and $\kappa$ is a constant, we conclude that the module $L$ of the angular momentum is conserved even though the vector $\boldsymbol{L}$ is not.

We can now use the remaining equation of motion (16) to obtain the conservation of the total energy of the system. To do so, it can be rewritten, in terms of $\boldsymbol{L}$ given by $(18)$, as

$$
\mu \ddot{r}-\frac{L^{2}}{\mu r^{3}}-\frac{1}{4 \pi \epsilon_{0}} \frac{q}{r^{2}}=0 .
$$

Now, to proceed further, we may do the trick [1, p. 74] of rewriting its right-hand side as a derivative in $r$ and multiplying both sides by $\dot{r}$ as

$$
\mu \ddot{r} \dot{r}=-\frac{\mathrm{d}}{\mathrm{d} r}\left(\frac{L^{2}}{2 \mu r^{2}}+\frac{1}{4 \pi \epsilon_{0}} \frac{q}{r}\right) \dot{r}
$$

from what, remembering that $\mathrm{d} f(r) / \mathrm{d} t=(\mathrm{d} r / \mathrm{d} t) \mathrm{d} f(r) / \mathrm{d} r=\dot{r} \mathrm{~d} f(r) / \mathrm{d} r$ and that $\mathrm{d} \dot{r}^{2} / \mathrm{d} t=2 \ddot{r} \dot{r}$, it follows that

$$
\frac{\mathrm{d}}{\mathrm{d} t}\left(\frac{\mu}{2} \dot{r}^{2}\right)=-\frac{\mathrm{d}}{\mathrm{d} t}\left(\frac{L^{2}}{2 \mu r^{2}}+\frac{1}{4 \pi \epsilon_{0}} \frac{q}{r}\right),
$$

which expresses the conservation of the total energy of the system

$$
E=\frac{\mu}{2} \dot{r}^{2}+\frac{L^{2}}{2 \mu r^{2}}+\frac{1}{4 \pi \epsilon_{0}} \frac{q}{r} .
$$

Notice, now, that the definitions (22) of the $\boldsymbol{J}$ vector and (18) of the angular momentum $\boldsymbol{L}$ lead to the result

$$
\begin{aligned}
\boldsymbol{J} \cdot \hat{\boldsymbol{r}} & =\left(\mu \boldsymbol{r} \times \boldsymbol{v}+\frac{\mu_{0}}{4 \pi} \kappa \hat{\boldsymbol{r}}\right) \cdot \hat{\boldsymbol{r}} \\
& =\frac{\mu_{0}}{4 \pi} \kappa
\end{aligned}
$$

where we used the fact that $\boldsymbol{r} \times \boldsymbol{v} \cdot \hat{\boldsymbol{r}}=0$. 
If we interpret $\boldsymbol{J} \cdot \hat{\boldsymbol{r}}$ as the projection of $\boldsymbol{J}$ on the direction of $\hat{\boldsymbol{r}}$, we can define $\alpha$ as the angle formed by $\hat{\boldsymbol{r}}$ and $\boldsymbol{J}$ given by

$$
\cos \alpha \equiv \frac{\boldsymbol{J} \cdot \hat{\boldsymbol{r}}}{J}
$$

which, as we see from $(25)$ and $(22)$, is constant, with value

$$
\alpha=\arccos \left(\mu_{0} \kappa / 4 \pi J\right)
$$

or, by using the trigonometric identity

$$
\tan (\arccos x)=\frac{\sqrt{1-x^{2}}}{x}
$$

we get from 26 and 23

$$
\begin{aligned}
\alpha & =\arctan \left(\frac{\sqrt{1-\left(\mu_{0} \kappa / 4 \pi J\right)^{2}}}{\mu_{0} \kappa / 4 \pi J}\right) \\
& =\arctan \left(\frac{4 \pi J \sqrt{1-\left(\mu_{0} \kappa / 4 \pi J\right)^{2}}}{\mu_{0} \kappa}\right) \\
& =\arctan \left(\frac{4 \pi \sqrt{J^{2}-\left(\mu_{0} / 4 \pi\right)^{2} \kappa^{2}}}{\mu_{0} \kappa}\right) \\
& =\arctan \left(4 \pi \sqrt{L^{2}} / \mu_{0} \kappa\right) \\
& =\arctan \left(4 \pi L / \mu_{0} \kappa\right) .
\end{aligned}
$$

Now, being $\boldsymbol{J}$ a vector fixed in the space and the angle it forms with $\hat{\boldsymbol{r}}$ constant, it implies that the motion is limited to the surface of a cone (the Poincaré cone) (Figure 2 ) of constant half-aperture angle $\alpha$ given by

$$
\alpha=\arccos \left(\mu_{0}|\kappa| / 4 \pi J\right),
$$

with $\boldsymbol{J}$ being coincident with the interior axis if $\kappa$ is positive and with its exterior axis if $\kappa$ is negative. A similar conclusion was obtained by Poincaré [6] for the movement of an electric charge in the field of a pole of a magnet (equivalent to a Dirac's monopole) and Appel [16] for the movement of a electric charge in the field of a magnetic and electric pole simultaneously (equivalent to a Schwinger's dyon).

The fact that the motion is limited to the surface of a cone of constant half-aperture angle $\alpha$ allows us to choose a new spherical coordinate system $(r, \alpha, \beta)$, in which the vector $\boldsymbol{J}$ coincides with the polar axis, so as to have only the two degrees of freedom radial distance $(r)$ and azimuthal angle $(\beta)$.

With this coordinate system, $\dot{\alpha}=0$, while the Lagrangian 17 reduces to

$$
\mathscr{L}=\frac{1}{2} \mu \dot{r}^{2}+\frac{1}{2} \mu r^{2} \sin ^{2} \alpha \dot{\beta}^{2}-\frac{1}{4 \pi \epsilon_{0}} \frac{q}{r}+\frac{\mu_{0}}{4 \pi} \kappa \cos \alpha \dot{\beta}
$$

and the equations of motion 16 result

$$
\begin{aligned}
& \mu \ddot{r}-\mu r \sin ^{2} \alpha \dot{\beta}^{2}-\frac{1}{4 \pi \epsilon_{0}} \frac{q}{r^{2}}=0 \\
& \mu r^{2} \sin \alpha \cos \alpha \dot{\beta}^{2}=\frac{\mu_{0}}{4 \pi} \kappa \sin \alpha \dot{\beta} \\
& 2 \mu r \dot{r} \sin ^{2} \alpha \dot{\beta}^{2}+\mu r^{2} \sin ^{2} \alpha \ddot{\beta}=0 .
\end{aligned}
$$


In the same way, the angular momentum $(18)$ is now expressed by

$$
\boldsymbol{L}=-\mu r^{2} \sin \alpha \dot{\beta} \hat{\boldsymbol{\alpha}},
$$

the conserved module of the angular momentum as

$$
L=\mu r^{2} \sin \alpha \dot{\beta},
$$

and the conserved energy $(24)$ results

$$
E=\frac{\mu \dot{r}^{2}}{2}+\frac{L^{2}}{2 \mu r^{2}}+\frac{1}{4 \pi \epsilon_{0}} \frac{q}{r} .
$$

Now, to arrive at the equation of the orbit, we will follow Goldstein's procedure [1, sec. 3.7].

To start with, we can solve (33) for $\dot{r}$ and get

$$
\dot{r}=\sqrt{\frac{2}{\mu}\left(E-\frac{1}{4 \pi \epsilon_{0}} \frac{q}{r}-\frac{L^{2}}{2 \mu r^{2}}\right)}
$$

or, being $\dot{r}=\mathrm{d} r / \mathrm{d} t$,

$$
\mathrm{d} t=\left(\frac{2 E}{\mu}-\frac{q}{2 \pi \epsilon_{0} \mu r}-\frac{L^{2}}{\mu^{2} r^{2}}\right)^{-1 / 2} \mathrm{~d} r .
$$

For the equation of the orbit, we need the dependence of $r$ upon $\theta$ eliminating the parameter $t$. This elimination can be done by seeing (32) as a relation between $\mathrm{d} \beta$ and $\mathrm{d} t$, in the same way as we $\operatorname{did}$ for $\dot{r}$ :

$$
L \mathrm{~d} t=\mu r^{2} \sin \alpha \mathrm{d} \beta
$$

or

$$
\mathrm{d} \beta=\frac{L}{\mu r^{2} \sin \alpha} \mathrm{d} t .
$$

The substitution of (35) into (37) yields

$$
\mathrm{d} \beta=\frac{L}{\mu r^{2} \sin \alpha}\left(\frac{2 E}{\mu}-\frac{q}{2 \pi \epsilon_{0} \mu r}-\frac{L^{2}}{\mu^{2} r^{2}}\right)^{-1 / 2} \mathrm{~d} r .
$$

Now, integrating (38) after slight rearrangements, we obtain

$$
\beta=\frac{1}{\sin \alpha} \int\left(\frac{2 \mu E}{L^{2}}-\frac{\mu q}{2 \pi \epsilon_{0} L^{2} r}-\frac{1}{r^{2}}\right)^{-1 / 2} \frac{\mathrm{d} r}{r^{2}}+\beta^{\prime},
$$

where $\beta^{\prime}$ is a constant of integration determined by the initial conditions and not necessarily being the same as the initial angle $\beta_{0}$ at time $t=0$.

Finally, changing the variable of integration to $u=1 / r$, we obtains

$$
\beta=\beta^{\prime}-\frac{1}{\sin \alpha} \int\left(\frac{2 \mu E}{L^{2}}-\frac{\mu q}{2 \pi \epsilon_{0} L^{2}} u-u^{2}\right)^{-1 / 2} \mathrm{~d} u .
$$

This indefinite integral is of the standard form [1, p. 93]

$$
\int \frac{\mathrm{d} x}{\sqrt{a+b u+c u^{2}}}=\frac{1}{\sqrt{-c}} \arccos \left(-\frac{b+2 c u}{\sqrt{\Delta}}\right),
$$


where

$$
\Delta=b^{2}-4 a c,
$$

where we identify

$$
\begin{aligned}
& a=2 \mu E / L^{2}, \\
& b=-\mu q / 2 \pi \epsilon_{0} L^{2}, \text { and } \\
& c=-1 .
\end{aligned}
$$

Applying (43) to (42), we obtain

$$
\begin{aligned}
\Delta & =b^{2}\left(1-4 a c / b^{2}\right) \\
& =\left(\frac{\mu q}{2 \pi \epsilon_{0} L^{2}}\right)^{2}\left[1+4 \frac{2 \mu E}{L^{2}}\left(\frac{2 \pi \epsilon_{0} L^{2}}{\mu q}\right)^{2}\right] \\
& =\left(\frac{\mu q}{2 \pi \epsilon_{0} L^{2}}\right)^{2}\left[1+\frac{2\left(4 \pi \epsilon_{0}\right)^{2} E L^{2}}{\mu q^{2}}\right]
\end{aligned}
$$

while applying (43) to $b+2 c x$ results

$$
\begin{aligned}
b+2 c x & =b(1+2 c / b x) \\
& =\left(\frac{\mu q}{2 \pi \epsilon_{0} L^{2}}\right)\left(1-2 \frac{2 \pi \epsilon_{0} L^{2}}{\mu q} u\right) .
\end{aligned}
$$

Now inserting these results into 40 , we obtains

$$
\beta=\beta^{\prime}-\frac{1}{\sin \alpha} \arccos \left[\left(\frac{4 \pi \epsilon_{0} L^{2} u}{\mu q}-1\right) / \sqrt{1+\frac{2\left(4 \pi \epsilon_{0}\right)^{2} E L^{2}}{\mu q^{2}}}\right]
$$

which we can solve for $u=1 / r$, obtaining the equation of the orbit as

$$
\frac{1}{r}=-\frac{\mu q}{4 \pi \epsilon_{0} L^{2}}\left\{1+\sqrt{1+\frac{32 \pi^{2} \epsilon_{0}^{2} E L^{2}}{\mu q^{2}}} \cos \left[\sin \alpha\left(\beta-\beta^{\prime}\right)\right]\right\},
$$

where we now identify $\beta^{\prime}$ as one of the turning angles of the orbit.

One sees that, except for the $\sin \alpha$ term, 47) is very similar to the equation for the Kepler problem of the planetary orbits [1, p. 93]. As a matter of fact, if the cone has been degenerated to a plane $(\alpha=\pi / 2)$, the equation of the orbit (47) results

$$
\frac{1}{r}=-\frac{\mu q}{4 \pi \epsilon_{0} L^{2}}\left[1+\varepsilon \cos \left(\beta-\beta^{\prime}\right)\right]
$$

which represents a conic curve with eccentricity

$$
\varepsilon=\sqrt{1+32 \pi^{2} \epsilon_{0}^{2} E L^{2} / \mu q^{2}}
$$

Now, remembering that the distance between two points that are differentially separated on the surface of a cone with a half angle $\alpha$ is

$$
(\mathrm{d} s)^{2}=(\mathrm{d} r)^{2}+r^{2}(\sin \alpha \mathrm{d} \beta)^{2},
$$

while the distance between two points on the plane is given by

$$
(\mathrm{d} s)^{2}=(\mathrm{d} r)^{2}+r^{2}(\mathrm{~d} \phi)^{2},
$$




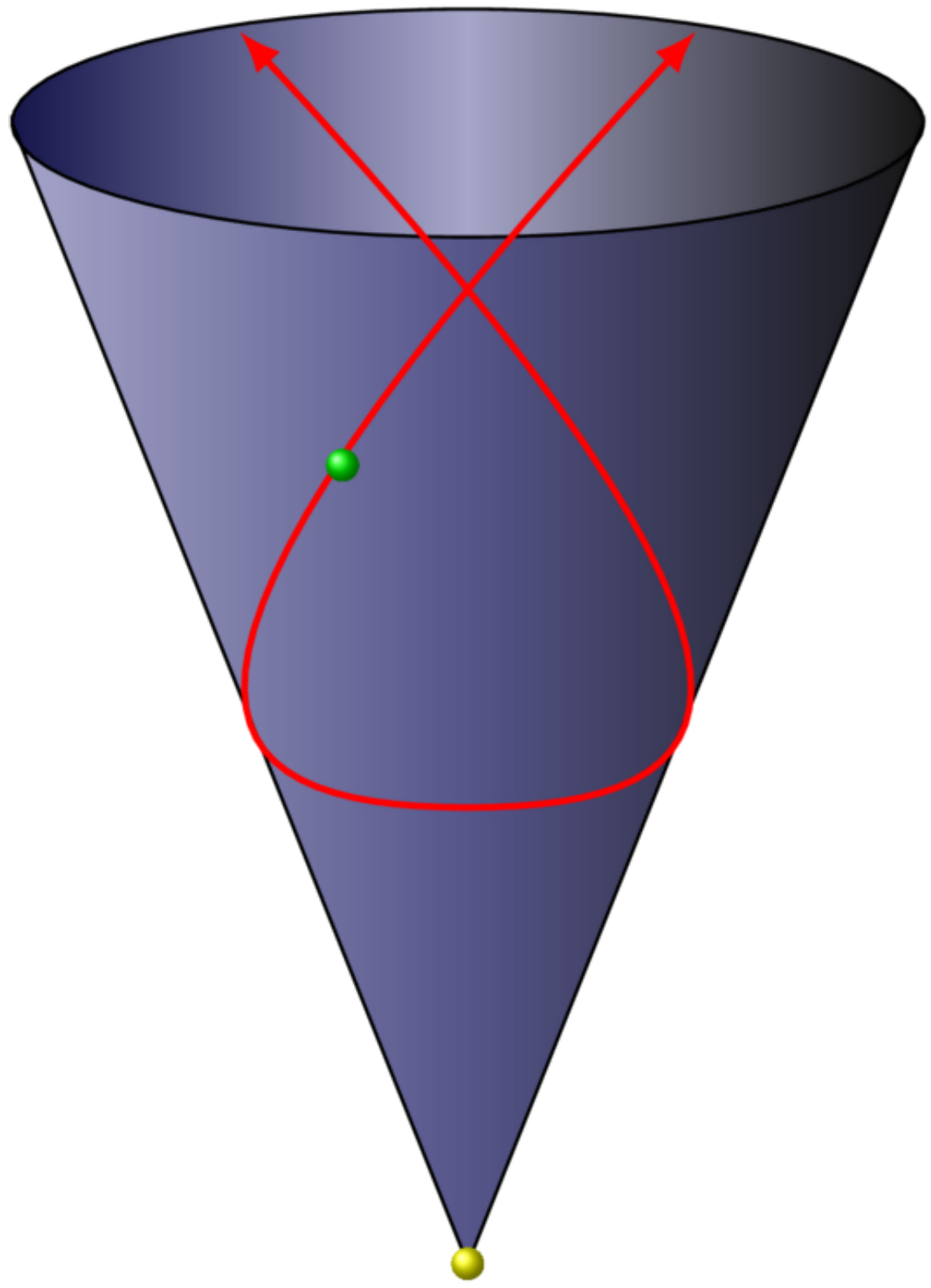

Figure 2. The orbit of the dyon confined to the surface of the Poincare cone with half-aperture angle $\alpha$, according to (71).

we interpret (47) as representing a conic-shaped orbit confined to the surface of the Poincaré cone, as shown in Figure 2.

As in Kepler problem, such conic-shaped orbits divide themselves into bound states with elliptic-like orbits $(\varepsilon<1)$ and scatterings with hyperbolic-like orbits $(\varepsilon>1)[1$, p. 94]. We will explore both cases in the next two sections. 


\section{Bound dyon-dyon states}

Let us consider first the bound states $(\varepsilon<1)$ of the dyon-dyon system.

From 49, this corresponds to

$$
\sqrt{1+32 \pi^{2} \epsilon_{0}^{2} E L^{2} / \mu q^{2}}<1
$$

or

$$
\begin{aligned}
& 1+32 \pi^{2} \epsilon_{0}^{2} E L^{2} / \mu q^{2}<1 \\
& 32 \pi^{2} \epsilon_{0}^{2} E L^{2} / \mu q^{2}<0
\end{aligned}
$$

which, being everything else positive, implies

$$
E<0
$$

as expected.

Now, from (33), (52) implies

$$
\begin{aligned}
& \frac{\mu \dot{r}^{2}}{2}+\frac{L^{2}}{2 \mu r^{2}}+\frac{1}{4 \pi \epsilon_{0}} \frac{q}{r}<0 \\
& \frac{1}{4 \pi \epsilon_{0}} \frac{q}{r}<\frac{\mu \dot{r}^{2}}{2}+\frac{L^{2}}{2 \mu r^{2}}
\end{aligned}
$$

which again, being everything else positive, implies

$$
q<0,
$$

i.e., an attractive interaction with

$$
\frac{1}{4 \pi \epsilon_{0}} \frac{|q|}{r}>\frac{\mu \dot{r}^{2}}{2}+\frac{L^{2}}{2 \mu r^{2}} \text {. }
$$

Being elliptic-like orbits, we can calculate, from (47), its two turning points (points of least or greatest distance of one dyon from the other), $r_{\min }$ and $r_{\max }$, as

$$
\begin{aligned}
& r_{\text {min }}=\frac{4 \pi \epsilon_{0} L^{2}}{\mu|q|}(1+\varepsilon) \\
& r_{\max }=\frac{4 \pi \epsilon_{0} L^{2}}{\mu|q|}(1-\varepsilon)
\end{aligned},
$$

from which we can obtain the semi-major axis $a$ and semi-minor axis $b$ as

$$
\begin{aligned}
& a=\frac{r_{\min }+r_{\max }}{2} \\
& b=\sqrt{a^{2}\left(1-\varepsilon^{2}\right)} .
\end{aligned}
$$

However, it is more useful to have $a$ and $b$ expressions in terms of the energy of the system. To start with, we make use of the fact that the radial velocity $\dot{r}$ is zero at those turning points. Therefore, from (33), the conserved energy at those points becomes

$$
E=\frac{L^{2}}{2 \mu r^{2}}+\frac{1}{4 \pi \epsilon_{0}} \frac{q}{r}
$$


which can be rewritten in the form of a quadratic equation as

$$
r^{2}-\frac{1}{4 \pi \epsilon_{0}} \frac{q}{E} r-\frac{L^{2}}{2 \mu E}=0
$$

having the turning points $r_{\min }$ and $r_{\max }$ as its roots. Now, it is well known that the sum of the roots of a quadratic equation equals the negative of the coefficient of its linear term. Therefore,

$$
r_{\min }+r_{\max }=\frac{1}{4 \pi \epsilon_{0}} \frac{q}{E},
$$

and we obtain the semi-major axis $a$ as

$$
a=\frac{r_{\min }+r_{\max }}{2}=\frac{1}{8 \pi \epsilon_{0}} \frac{q}{E}
$$

and the semi-minor axis $b$ as

$$
\begin{aligned}
b & =\sqrt{a^{2}\left(1-\varepsilon^{2}\right)} \\
& =\sqrt{\left(\frac{1}{8 \pi \epsilon_{0}} \frac{q}{E}\right)^{2}\left[1-\left(1+\frac{32 \pi^{2} \epsilon_{0}^{2} E L^{2}}{\mu q^{2}}\right)\right]} \\
& =\sqrt{\left(\frac{q^{2}}{64 \pi^{2} \epsilon_{0}^{2} E^{2}}\right)\left(\frac{32 \pi^{2} \epsilon_{0}^{2} E L^{2}}{\mu q^{2}}\right)} \\
& =\frac{L}{\sqrt{2 \mu|E|}} .
\end{aligned}
$$

Notice that the existence of $r_{\min }$ and $r_{\max }$ does not necessarily mean that the orbit is closed but only that it is 'bounded' by those limiting distances.

To analyse the closedness of the orbits, we have to see that the term $\cos \left[\sin \alpha\left(\beta-\beta^{\prime}\right)\right]$ in $(47)$ implies that the ratio between the periodicities of the radial coordinate $(r)$ and the azimuthal angle $(\beta)$ is given by $\sin \alpha$. Consequently, it is this parameter that will determine if the orbit is closed or not.

To understand that, we have to consider that, at each revolution, the dyon describes a portion $\sin \alpha$ of the ellipse. Now, the orbit will be closed if, say, after $n$ revolutions, it will have completed exactly $m$ ellipses, that is, if and only if $\sin \alpha$ is rational

$$
\sin \alpha=\frac{m}{n},
$$

with $m$ and $n$ relatively prime numbers and $m \leq n$ [1, p. 91].

Furthermore, the numbers $m$ and $n$ define the topology of the orbits in terms of number of double points [14, p. 28], classifying them into families of the same topology.

\section{Dyon-dyon scattering}

After the dyon-dyon bound states, seen in the previous section, let us now study the classical scattering $(\varepsilon>1)$ of one dyon by another.

For this study, we need to obtain the so-called cross-section for scattering in a given direction $\sigma(\Omega)$ and to arrive at it we will follow Goldstein's procedure [1, sec. 3.10]. 
From (49) and a reasoning similar to the one that leaded to (52), this corresponds to

$$
E>0
$$

which, from (33), analogously to (53), implies a repulsive interaction, i.e.

$$
q>0 .
$$

To start with, let us define $v_{0}$ as the velocity of the dyon when it is at an infinite distance $(r \rightarrow \infty)$ from the other. Then, from (33), the conserved energy reduces to the kinetic energy at that point:

$$
E=\frac{1}{2} \mu v_{0}^{2}
$$

We can now define $d$ as the distance of closest approach (periapsis) and impose the following condition on $\beta^{\prime}$

$$
\beta^{\prime}=\beta(r=d)=0
$$

into the general equation of the orbit 47 , obtaining

$$
\frac{1}{r}=-\frac{\mu q}{4 \pi \epsilon_{0} L^{2}}[1+\varepsilon \cos (\beta \sin \alpha)]
$$

with $\varepsilon$ now given by substituting (60) into 49 as

$$
\begin{aligned}
\varepsilon & =\sqrt{1+32 \pi^{2} \epsilon_{0}^{2}\left(\mu v_{0}^{2} / 2\right) L^{2} / \mu q^{2}} \\
& =\sqrt{1+\left(4 \pi \epsilon_{0}\right)^{2} L^{2} v_{0}^{2} / q^{2}},
\end{aligned}
$$

a result similar to that obtained by Schwinger [17.

We can now study the dyon-dyon scattering by defining the scattering angle $\Theta$, the angle between incidence $\hat{\boldsymbol{r}}_{i}$ and scattering $\hat{\boldsymbol{r}}_{f}$ directions at an infinite distance from the origin, as

$$
\cos \Theta=-\hat{\boldsymbol{r}}_{i} \cdot \hat{\boldsymbol{r}}_{f} .
$$

Expressing $\hat{\boldsymbol{r}}$ in the coordinate system $(r, \alpha, \beta)$ by

$$
\hat{\boldsymbol{r}}=\sin \alpha \cos \beta \hat{\boldsymbol{\imath}}+\sin \alpha \sin \beta \hat{\boldsymbol{j}}+\cos \alpha \hat{\boldsymbol{k}}
$$

with $\alpha$ constant and given by (28), we obtain, from (64),

$$
\cos \Theta=-\left[\sin ^{2} \alpha\left(\cos \beta_{i} \cos \beta_{f}+\sin \beta_{i} \sin \beta_{f}\right)+\cos ^{2} \alpha\right],
$$

where $\beta_{i}$ and $\beta_{f}$ are the azimuthal angles of the directions of incidence and scattering at an infinite distance from the origin, respectively, and, by making use of a few trigonometric identities such as

$$
\begin{aligned}
& \cos ^{2} \theta=1-\sin ^{2} \theta \\
& \cos \left(\theta_{1}-\theta_{2}\right)=\cos \theta_{1} \cos \theta_{2}+\sin \theta_{1} \cos \theta_{2} \\
& \sin ^{2}(\theta / 2)=\frac{1-\cos \theta}{2} \\
& \cos (\theta / 2)= \pm \sqrt{(1+\cos \theta) / 2}
\end{aligned}
$$


we obtain

$$
\begin{aligned}
\cos \Theta & =-\left[\sin ^{2} \alpha \cos \left(\beta_{i}-\beta_{f}\right)+\left(1-\sin ^{2} \alpha\right)\right] \text { by }(66 a) \text { and }(66 b) \\
& =\sin ^{2} \alpha\left[1-\cos \left(\beta_{i}-\beta_{f}\right)\right]-1 \\
& =\sin ^{2} \alpha\left\{2 \sin ^{2}\left[\left(\beta_{i}-\beta_{f}\right) / 2\right]\right\}-1 \quad \text { by }(66 c) \\
& =2\left\{\sin \alpha \sin \left[\left(\beta_{i}-\beta_{f}\right) / 2\right]\right\}^{2}-1,
\end{aligned}
$$

and, finally, by $(66 d)$,

$$
\cos (\Theta / 2)=\sin \alpha\left|\sin \left[\left(\beta_{i}-\beta_{f}\right) / 2\right]\right| .
$$

To proceed further, we need to calculate $\beta_{i}$ and $\beta_{f}$, the azimuthal angles of the directions of incidence and scattering at an infinite distance from the origin. Notice that $(1 / r)_{r \rightarrow \infty}=0$ and, therefore, from 62 , we get

$$
\left(-\frac{\mu q}{4 \pi \epsilon_{0} L^{2}}[1+\varepsilon \cos (\beta \sin \alpha)]\right)_{r \rightarrow \infty}=0
$$

and we can evaluate $\cos (\beta \sin \alpha)_{r \rightarrow \infty}$ as

$$
\begin{aligned}
\cos (\beta \sin \alpha)_{r \rightarrow \infty} & =-1 / \varepsilon \\
& =-\left[1+\left(4 \pi \epsilon_{0}\right)^{2} L^{2} v_{0}^{2} / q^{2}\right]^{-1 / 2} \\
& =-\left[1+\left(4 \pi \epsilon_{0}\right)^{2}\left(\mu_{0} \kappa \tan \alpha / 4 \pi\right)^{2} v_{0}^{2} / q^{2}\right]^{-1 / 2} \\
& =-\left(1+\left(\kappa^{2} v_{0}^{2} / c^{4} q^{2}\right) \tan ^{2} \alpha\right)^{-1 / 2}
\end{aligned}
$$

where we used (63), (27), and $\mu_{0} \epsilon_{0}=1 / c^{2}$.

Now, by making use of the trigonometric identity

$$
\cos (\arctan x)=\frac{1}{\sqrt{1+x^{2}}}
$$

into (68), we obtain

$$
\begin{aligned}
(\beta \sin \alpha)_{r \rightarrow \infty} & =\arctan \left[\left(\kappa v_{0} / c^{2}|q|\right) \tan \alpha\right] \\
& =\arctan (k \tan \alpha),
\end{aligned}
$$

where we introduced the parameter $k$ defined as

$$
k \equiv \kappa v_{0} / c^{2}|q| .
$$

Therefore, from (67), we finally obtain the scattering angle $\Theta$ as

$$
\cos (\Theta / 2)=\sin \alpha|\sin (\Phi / \sin \alpha)|,
$$

where, for compactness, we introduced the parameter $\Phi$ defined by

$$
\Phi \equiv(\beta \sin \alpha)_{r \rightarrow \infty}=\arctan (k \tan \alpha),
$$

from the result 69 .

We present in Figure 3 graphics of the functional dependence of $\Theta$ upon $\alpha$ for various values of the parameter $k$. In this figure, it is also displayed a graph for monopoleelectron scattering, obtained by making $e_{2}=g_{1}=0$ (which, from $(9$ ), results in $q=0$ and $\kappa=-e g / c)$ in $(69)$. 

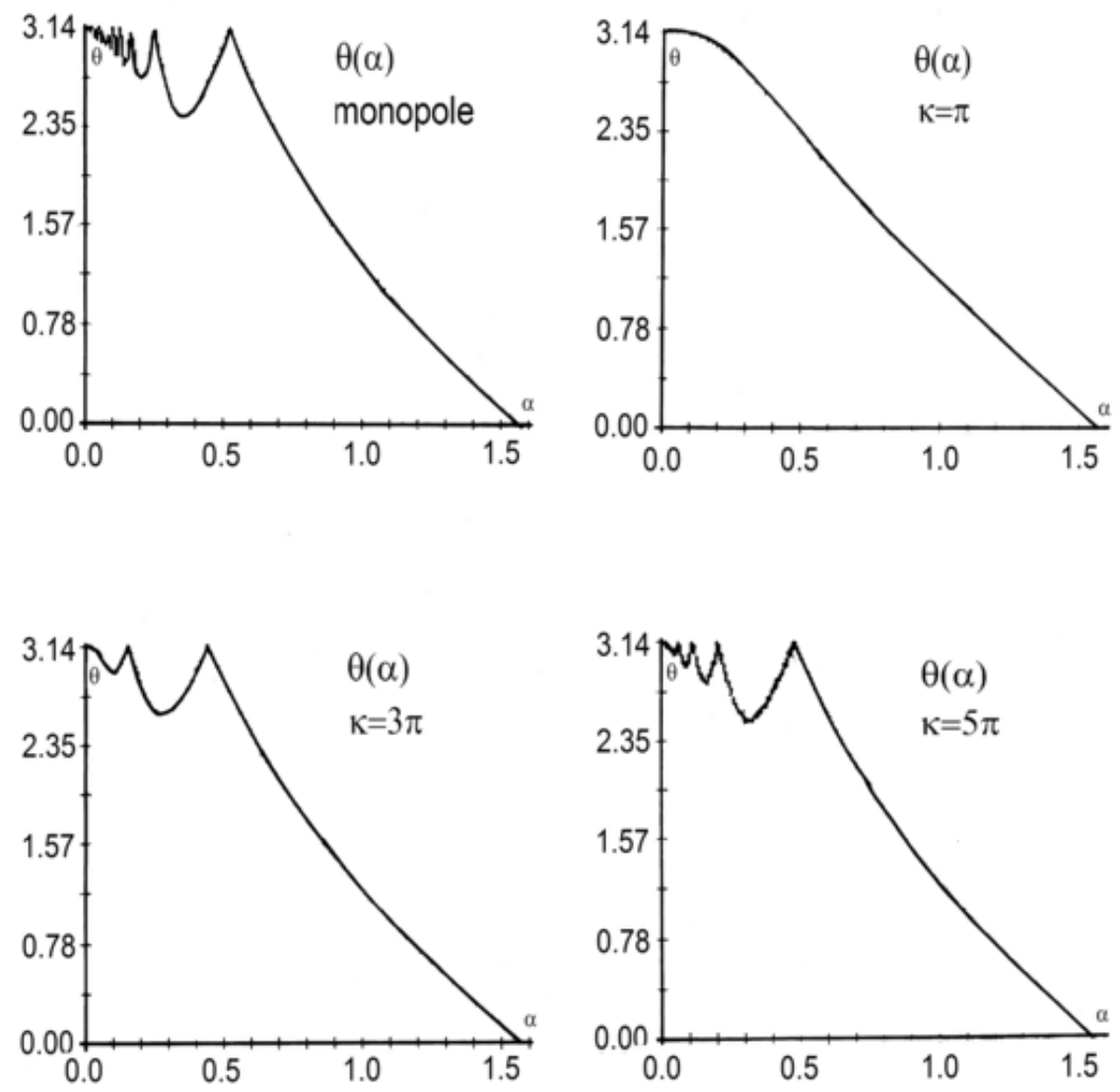

Figure 3. Graphs of the functional dependence of the scattering angle $\Theta$ upon the half-aperture angle of the cone, $\alpha$, according to (71), for the monopole-electron pair and for various values of the parameter $k \equiv \kappa v_{0} / c^{2} q$.

As the scattering orbit is asymptotic to $\hat{\boldsymbol{r}}_{i}$ and $\hat{\boldsymbol{r}}_{f}$, which define a plane, we can calculate the elastic differential cross-section [1, p. 108] for the dyon-dyon scattering, in terms of the impact parameter $s$ (the perpendicular distance between the centre of force and the incident velocity) [1, p. 107], by the equation

$$
\frac{\mathrm{d} \sigma}{\mathrm{d} \Omega}=\left|\frac{s \mathrm{~d} s}{d(\cos \Theta)}\right|=\sum_{s} s\left|\frac{\mathrm{d}(\cos \Theta)}{\mathrm{d} s}\right|^{-1}
$$

since, as seen from the graphs in Figure 3 , in general, $\alpha$ is a multivalued function of $\Theta[1$, p. 111].

As $v_{0}$ is the incident velocity and perpendicular to the impact parameter $s$, we can express the angular momentum $L$, from (18), as $L=\mu s v_{0}$ and also substitute (27), resulting

$$
\begin{aligned}
s & =L /\left(\mu v_{0}\right) \\
& =\left(\mu_{0} \kappa / 4 \pi \mu v_{0}\right) \tan \alpha,
\end{aligned}
$$

Now, using $\mathrm{d} f(\alpha) / \mathrm{d} s=(\mathrm{d} \alpha / \mathrm{d} s) \mathrm{d} f(\alpha) / \mathrm{d} \alpha=(\mathrm{d} s / \mathrm{d} \alpha)^{-1} \mathrm{~d} f(\alpha) / \mathrm{d} \alpha$ and $\tan ^{\prime} \theta=$ 
$\sec ^{2} \theta=\cos ^{-2} \theta$, we can rewrite $(73)$ as a relation between $\mathrm{d} s$ and $\mathrm{d} \alpha$ as

$$
\frac{\mathrm{d}}{\mathrm{d} s}=\left[\left(\frac{\mu_{0} \kappa}{4 \pi \mu v_{0}}\right) \frac{1}{\cos ^{2} \alpha}\right]^{-1} \frac{\mathrm{d}}{\mathrm{d} \alpha},
$$

and use this and $(73)$ to evaluate the derivatives with respect to $s$ in $(72)$, obtaining the elastic differential cross-section as

$$
\begin{aligned}
\frac{\mathrm{d} \sigma}{\mathrm{d} \Omega} & =\sum_{\alpha}\left[\left(\frac{\mu_{0} \kappa}{4 \pi \mu v_{0}}\right) \frac{1}{\cos ^{2} \alpha}\right]\left|\frac{\mathrm{d}(\cos \Theta)}{\mathrm{d} \alpha}\right|^{-1}\left[\left(\frac{\mu_{0} \kappa}{4 \pi \mu v_{0}}\right) \tan \alpha\right] \\
& =\sum_{\alpha}\left(\frac{\mu_{0} \kappa}{4 \pi \mu v_{0}}\right)^{2} \frac{\sin \alpha}{\cos ^{3} \alpha}\left|\frac{\mathrm{d}(\cos \Theta)}{\mathrm{d} \alpha}\right|^{-1} \\
& =\sum_{\alpha}\left(\frac{\mu_{0} \kappa}{4 \pi \mu v_{0}}\right)^{2} \frac{1}{2 \cos ^{4} \alpha}\left|\frac{\sin (2 \alpha)}{\sin \Theta} \frac{\mathrm{d} \alpha}{\mathrm{d} \Theta}\right|,
\end{aligned}
$$

where, in the last step, we used the trigonometric identity $\sin (2 \alpha)=2 \sin \alpha \cos \alpha$.

Particularly interesting are the cases where the parameter $k$ defined in 70 is a multiple of $\pi$. One can see that by expanding (72) with (69) around $\alpha=0$, using the approximations $\tan \theta \cong \sin \theta$ and $\arctan \theta \cong \theta$, as

$$
\begin{aligned}
\Phi & =\arctan \left[\left(\kappa v_{0} / c^{2}|q|\right) \tan \alpha\right] \\
& \cong\left(\kappa v_{0} / c^{2}|q|\right) \sin \alpha,
\end{aligned}
$$

and using this result in 71 , obtaining

$$
\begin{aligned}
\cos (\Theta / 2) & \cong \sin \alpha\left|\sin \left\{\left[\left(\kappa v_{0} / c^{2}|q|\right) \sin \alpha\right] / \sin \alpha\right\}\right| \\
& \cong \alpha\left|\sin \left(\kappa v_{0} / c^{2}|q|\right)\right|,
\end{aligned}
$$

from which, by means of the trigonometric identity $\cos (2 \theta)=2 \cos ^{2} \theta-1$, we obtain

$$
\cos (\Theta) \cong 2 \alpha^{2} \sin ^{2}\left(\kappa v_{0} / c^{2}|q|\right)-1
$$

With these approximations and the usual $\sin \theta \cong \theta$ and $\cos \theta \cong 1$ ones, the crosssection 75 results

$$
\begin{aligned}
& \frac{\mathrm{d} \sigma}{\mathrm{d} \Omega} \stackrel{\alpha \rightarrow 0}{\rightarrow} \sum_{\alpha}\left(\frac{\mu_{0} \kappa}{4 \pi \mu v_{0}}\right)^{2} \alpha\left|\frac{\mathrm{d}\left[2 \alpha^{2} \sin ^{2}\left(\kappa v_{0} / c^{2}|q|\right)-1\right]}{\mathrm{d} \alpha}\right|^{-1} \\
& \stackrel{\alpha \rightarrow 0}{\rightarrow} \sum_{\alpha}\left(\frac{\mu_{0} \kappa}{4 \pi \mu v_{0}}\right)^{2} \alpha\left|4 \alpha \sin ^{2}\left(\kappa v_{0} / c^{2}|q|\right)\right|^{-1} \\
& \stackrel{\alpha \rightarrow 0}{\rightarrow}\left(\frac{\mu_{0} \kappa}{8 \pi \mu v_{0}}\right)^{2} \frac{1}{\sin ^{2}\left(\kappa v_{0} / c^{2}|q|\right)}
\end{aligned}
$$

and, therefore, when $\alpha \rightarrow 0$, a second order pole occur in the cross-section whenever

$$
k=n \pi \quad(n=1,2,3, \cdots) .
$$

To evaluate the differential cross-section 75 , we found it convenient to introduce the new variable

$$
\zeta \equiv 2 \Phi / \sin \alpha
$$


where $\Phi$ is given by $(72$ and 69 .

Now, from (71) and again by means of the trigonometric identity $\cos (2 \theta)=$ $2 \cos ^{2} \theta-1$, we have

$$
\cos (\Theta)=2 \sin \alpha^{2} \sin ^{2}(\zeta / 2)-1 .
$$

From this result, we can evaluate the derivative on the right-hand side of (75) as

$$
\begin{aligned}
\left(\frac{1}{\sin \Theta} \frac{\mathrm{d} \alpha}{\mathrm{d} \Theta}\right)^{-1} & =\frac{\mathrm{d} \cos \Theta}{\mathrm{d} \alpha} \\
& =\sin (2 \alpha)\left[(1-\cos \zeta)+\sin \zeta \tan \alpha \frac{\mathrm{d}(\zeta / 2)}{\mathrm{d} \alpha}\right] .
\end{aligned}
$$

Then, from 80 and 72 , we can evaluate the derivative of $\zeta / 2$ as

$$
\frac{\mathrm{d}(\zeta / 2)}{\mathrm{d} \alpha}=\frac{\kappa v_{0}}{q \sin \alpha} \frac{\cos ^{2}[(\zeta / 2) \sin \alpha]}{\cos ^{2} \alpha}-(\zeta / 2) \cot \alpha
$$

and obtain, from (75), the differential cross-section as

$$
\frac{\mathrm{d} \sigma}{\mathrm{d} \Omega}=\left(\frac{\mu_{0} \kappa}{4 \pi \mu v_{0}}\right)^{2} g(\zeta)
$$

where

$$
g(\zeta)=\sum_{\alpha} \frac{1}{\cos ^{4} \alpha} \frac{1}{|2(1-\cos \zeta)-\zeta \sin \zeta+(\sin \zeta / \sin \alpha) \sin (\zeta \sin \alpha)|},
$$

with $\zeta$ given by 80 .

In Figure 4, we present graphs of the differential cross-section for various values of the parameter $k$. In this figure, we also show a graph for the electron-monopole scattering obtained, again, by making $q=0$ and $\kappa=-e g$ in 85 .

One observes from Figure 4 that the cross-section becomes infinite for some values of $\Theta$ and falls abruptly thereafter. From $(75)$, we see that it happens, besides $\Theta=0$, in one of the following cases

$$
\left\{\begin{array}{l}
\mathrm{d} \Theta / \mathrm{d} \alpha=0 \\
\Theta=\pi \quad \text { but } \sin (2 \alpha) \neq 0
\end{array}\right.
$$

This phenomenon is very similar to what occurs in the optical scattering of sunlight by raindrops and, because of this similarity, these two conditions are referred to as rainbow scattering [1, p. 111] and glory scattering [1, p. 114], respectively.

In the case of rainbow scattering, we have, from (71),

$$
\tan \left(\frac{\Phi_{\mathrm{r}}}{\sin \alpha_{\mathrm{r}}}\right)-\frac{\Phi_{\mathrm{r}}}{\sin \alpha_{\mathrm{r}}}+\frac{\sin \left(2 \Phi_{\mathrm{r}}\right)}{\sin \left(2 \alpha_{\mathrm{r}}\right) \cos \alpha_{\mathrm{r}}}=0
$$

where the suffix $r$ was appended to indicate that it refers to the rainbow scattering. Equation (87) can be solved numerically. In Table 1, we present rainbow $\alpha_{\mathrm{r}}$ angles for two values of the $k$ parameter defined in (70).

For the glory scattering, we have, from (71),

$$
\Phi_{\mathrm{g}} / \sin \alpha_{\mathrm{g}}=n \pi \quad(n=1,2,3, \cdots),
$$



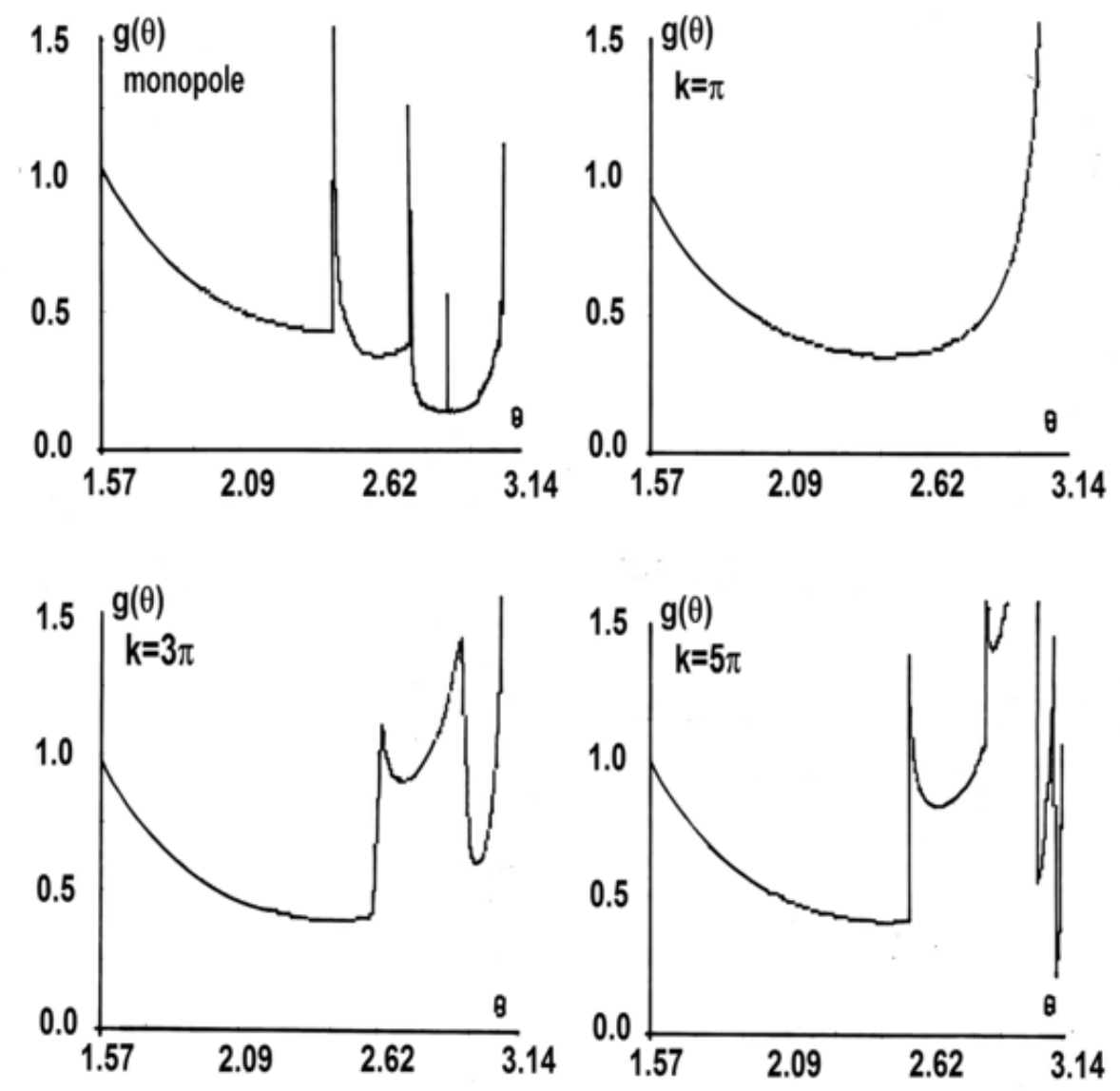

Figure 4. Differential cross-section $g(\Theta)$ scattering, according to 85 , for the monopole-electron pair and for various values of the parameter $k \equiv \kappa v_{0} / c^{2}|q|$.

Table 1. Rainbow angles $\alpha_{\mathrm{r}}(\mathrm{d} \Theta / \mathrm{d} \alpha=0)$ obtained from (87).

\begin{tabular}{ll}
\hline$k$ & $\alpha_{\mathrm{r}}$ \\
\hline $2 \pi$ & 0.230 \\
\hline $3 \pi$ & 0.104 \\
& 0.280 \\
\hline
\end{tabular}

and, from (72), the condition

$$
\tan \left(n \pi \sin \alpha_{\mathrm{g}}\right)=k \tan \alpha_{\mathrm{g}} \quad(n=1,2,3, \cdots),
$$

which can be solved numerically for the desired value of the parameter $k$. In Table 2 , we present glory $\alpha_{\mathrm{g}}$ angles for two values of the parameter $k$.

Let us now consider what happens at small-angle scattering $(\alpha \rightarrow \pi / 2)$. From (71) 
Table 2. Glory angles $\alpha_{\mathrm{g}}(\Theta=\pi)$ obtained from 89.

\begin{tabular}{ll}
\hline$k$ & $\alpha_{\mathrm{g}}$ \\
\hline $2 \pi$ & 0.394 \\
\hline $3 \pi$ & 0.156 \\
& 0.445 \\
\hline
\end{tabular}

and using the trigonometric identity $\sin \arctan \theta=x / \sqrt{1+x^{2}}=1 / \sqrt{1+x^{-2}}$, we have

$$
\begin{aligned}
\cos (\Theta / 2) & =\sin \alpha|\sin [\arctan (k \tan \alpha)]| \\
& =\frac{\sin \alpha}{\sqrt{1+(k \tan \alpha)^{-2}}} .
\end{aligned}
$$

Now, using (27) and expressing the parameter $k$ in terms of the impact parameter $s$ by means of $(73)$, we have

$$
\begin{aligned}
\tan \alpha & =\left(4 \pi / \mu_{0} \kappa\right) L \\
& =\left(4 \pi / \mu_{0} \kappa\right)\left(\mu v_{0} s\right) \\
& =\left(4 \pi \mu v_{0} / \mu_{0} \kappa\right) s,
\end{aligned}
$$

and, by means of the trigonometric identity $\sin \theta=1 / \sqrt{1+\cot ^{2} \theta}$, we obtain

$$
\sin \alpha=1 / \sqrt{1+\left(\mu_{0} \kappa / 4 \pi \mu v_{0} s\right)^{2}} .
$$

On the other hand, from (70) and (91), we get

$$
\begin{aligned}
k \tan \alpha & =\left(\kappa v_{0} / c^{2}|q|\right)\left(4 \pi \mu v_{0} / \mu_{0} \kappa\right) s \\
& =\left(4 \pi \epsilon_{0} \mu v_{0}^{2} s /|q|\right),
\end{aligned}
$$

where we used $\mu_{0} \epsilon_{0}=1 / c^{2}$ once more, and, therefore,

$$
\frac{1}{\sqrt{1+(k \tan \alpha)^{-2}}}=\frac{1}{\sqrt{1+\left(q / 4 \pi \epsilon_{0} \mu v_{0}^{2} s\right)^{2}}} .
$$

Substituting (92) and 94) into (90), using the approximation $1 / \sqrt{1+x} \cong 1-x / 2$, and keeping terms only up to first order, we obtain

$$
\begin{aligned}
\cos (\Theta / 2) & =\frac{1}{\sqrt{1+\left(q / 4 \pi \epsilon_{0} \mu v_{0}^{2} s\right)^{2}}} \frac{1}{\sqrt{1+\left(\mu_{0} \kappa / 4 \pi \mu v_{0} s\right)^{2}}} \\
& \cong\left[1-\frac{1}{2}\left(\frac{q}{4 \pi \epsilon_{0} \mu v_{0}^{2} s}\right)^{2}\right]\left[1-\frac{1}{2}\left(\frac{\mu_{0} \kappa}{4 \pi \mu v_{0} s}\right)^{2}\right] \\
& \cong 1-\frac{1}{2}\left[\left(\frac{q}{4 \pi \epsilon_{0} \mu v_{0}^{2} s}\right)^{2}+\left(\frac{\mu_{0} \kappa}{4 \pi \mu v_{0} s}\right)^{2}\right] \\
& \cong 1-\frac{1}{2} \frac{1}{\left(4 \pi \epsilon_{0} \mu v_{0}\right)^{2} s^{2}}\left[\left(\frac{q}{v_{0}}\right)^{2}+\left(\frac{\kappa}{c^{2}}\right)^{2}\right]
\end{aligned}
$$


Now, considering the approximation $\cos (\Theta / 2) \cong 1-(\Theta / 2)^{2} / 2$ and comparing this expression with (95), we have

$$
(\Theta / 2)^{2} \cong \frac{1}{\left(4 \pi \epsilon_{0} \mu v_{0}\right)^{2} s^{2}}\left[\left(\frac{q}{v_{0}}\right)^{2}+\left(\frac{\kappa}{c^{2}}\right)^{2}\right]
$$

or

$$
s^{2} \cong \frac{1}{(\Theta / 2)^{2}} \frac{1}{\left(4 \pi \epsilon_{0} \mu v_{0}\right)^{2}}\left[\left(\frac{q}{v_{0}}\right)^{2}+\left(\frac{\kappa}{c^{2}}\right)^{2}\right] .
$$

Thus, from $(72)$ and the approximation $\sin \theta \cong \theta$, we can compute the differential cross-section of the dyon-dyon scattering at small angles as

$$
\begin{aligned}
\frac{\mathrm{d} \sigma}{\mathrm{d} \Omega} & =s\left|\frac{\mathrm{d} s}{\mathrm{~d}(\cos \Theta)}\right| \\
& =s\left|\frac{\mathrm{d} s}{\mathrm{~d}\left(s^{2}\right)}\right|\left|\frac{\mathrm{d}\left(s^{2}\right)}{\mathrm{d}\left[(\Theta / 2)^{2}\right]}\right|\left|\frac{\mathrm{d}\left[(\Theta / 2)^{2}\right]}{\mathrm{d}(\Theta / 2)}\right|\left|\frac{\mathrm{d}(\Theta / 2)}{\mathrm{d}(\Theta)}\right|\left|\frac{\mathrm{d}(\Theta)}{\mathrm{d}(\cos \Theta)}\right| \\
& =s\left|\frac{\mathrm{d}\left(s^{2}\right)}{\mathrm{d} s}\right|^{-1}\left|\frac{\mathrm{d}\left[(\Theta / 2)^{2}\right]}{\mathrm{d}(\Theta / 2)}\right|\left|\frac{\mathrm{d}(\Theta / 2)}{\mathrm{d}(\Theta)}\right|\left|\frac{\mathrm{d}(\cos \Theta)}{\mathrm{d}(\Theta)}\right|^{-1}\left|\frac{\mathrm{d}\left(s^{2}\right)}{\mathrm{d}\left[(\Theta / 2)^{2}\right]}\right| \\
& =s|2 s|^{-1}|2(\Theta / 2)| \frac{1}{2}|-\sin \Theta|^{-1}\left|\frac{\mathrm{d}\left(s^{2}\right)}{\mathrm{d}\left[(\Theta / 2)^{2}\right]}\right| \\
& \cong \frac{1}{4} \frac{|\Theta|}{|\sin \Theta|} \frac{1}{\left(4 \pi \epsilon_{0} \mu v_{0}\right)^{2}}\left[\left(\frac{q}{v_{0}}\right)^{2}+\left(\frac{\kappa}{c^{2}}\right)^{2}\right]\left|\frac{\mathrm{d}}{\mathrm{d}\left[(\Theta / 2)^{2}\right]}\left(\frac{1}{(\Theta / 2)^{2}}\right)\right| \\
& \cong \frac{1}{4} \frac{1}{\left(4 \pi \epsilon_{0} \mu v_{0}\right)^{2}}\left[\left(\frac{q}{v_{0}}\right)^{2}+\left(\frac{\kappa}{c^{2}}\right)^{2}\right]\left|\frac{-1}{(\Theta / 2)^{4}}\right| \\
& \cong \frac{1}{4} \frac{1}{\left(4 \pi \epsilon_{0} \mu v_{0}\right)^{2}}\left[\left(\frac{q}{v_{0}}\right)^{2}+\left(\frac{\kappa}{c^{2}}\right)^{2}\right] \csc ^{4}(\Theta / 2)
\end{aligned}
$$

which is a generalization of the Rutherford formula for the scattering of $\alpha$ particles by atomic nuclei [1, p. 110].

A relativistic extension of the entire calculation previously done is possible, in a simple way [14, cap. 4]. The importance of a relativistic extension for the treatment of dyon-dyon system stems from the large value of the coupling constant for magnetic charges (see (3)). In the same way as in the non-relativistic case, the relativistic dyon is confined to the surface of a cone of half-aperture angle given by (27). The bound states correspond to conic-shaped orbits confined to the surface of the Poincaré cone (Figure 2), with a similar condition for the orbit closing. For the relativistic scattering case, the orbits are hyperbolas confined to the surface of the Poincaré cone and this system exhibits glory and rainbow scattering.

It is even possible to apply the well-known Sommerfeld (semi-classical) quantization rule [18, p. 283] to the dyon-dyon system. By doing it, we have shown the analogy between this system and the hydrogen atom and that the former can be considered its generalization [14, cap. 3]. Furthermore, we obtained, for both the non-relativistic 
and relativistic cases, an energy spectrum that reasonably approximates the quantum spectrum obtained by Pereira [19].

\section{Concluding remarks}

In this work, thanks to a Lagrangian without Dirac strings, it was possible to make a classical study of the dyon-dyon system, with the electron-monopole system as a particular case.

The orbit equations were interpreted as representing conic-shaped orbits confined to the surface of the Poincaré cone. While the electron does not form bound states with the magnetic monopole, the dyon-dyon system exhibits non-planar stable elliptic-like orbits, and we presented the conditions for them to be closed.

Through the orbit equations, it was also possible to study the classical scattering for these systems. We showed that the elastic differential cross-section shows divergences that are usually denominated glory and rainbow scatterings by their similarities with Optics. We also showed that the differential cross-section of the dyon-dyon scattering at small angles is a generalization of the Rutherford formula.

Finally, we want to stress that a relativistic extension of this study can be done in a simple way, as well as a semi-classical quantization via Sommerfeld rule.

We hope that these results may arouse students' and teachers' interest and contribute to Classical Mechanics courses.

\section{Acknowledgments}

We would like to thank the late Prof. Paulo Leal Ferreira, who was my adviser through the master's degree at the Institute for Theoretical Physics in São Paulo, where the thesis, on which this work is based, was done. We also acknowledge FAPESP (São Paulo Research Foundation) for a scholarship that made the M.Sc. research work possible.

\section{References}

[1] Goldstein H, Poole C and Safko J 2001 Classical Mechanics 3rd ed (San Francisco, CA: AddisonWesley)

[2] Sivardière J 2000 European Journal of Physics 21 183-190

[3] Dirac P A M 1931 Proceedings of the Royal Society of London. Series A, Mathematical and physical sciences 133 60-72

[4] Schwinger J 1969 Science 165 757-761

[5] Maxwell J C 1873 A Treatise on Electricity and Magnetism vol 2 (Oxford: Clarendon Press)

[6] Poincaré H 1896 Comptes rendus de l'Académie des Sciences 123 530-533

[7] Caruso F 1984 Sull'Origine della Condizione di Quantizzazione delle Cariche dei Dioni Tech. rep. Universitá di Torino Torino URL http://arxiv.org/abs/1308.3484

[8] Pinfold J L, Trache L, Smirnov A and Stoica S 2010 Dirac's Dream - the Search for the Magnetic Monopole Exotic Nuclei and Nuclear/Particle Astrophysics (III): From Nuclei to Stars - 23rd Carpathian Summer School of Physics, Sinaia, Romania, 20 Jun - 3 Jul, 2010 (American Institute of Physics) pp 234-239 
[9] Schwinger J 1966 Physical Review 144 1087-1093

[10] Yang C N 1977 Annals of New York Academy of Sciences 294 86-97

[11] Wu T T and Yang C N 1976 Nuclear Physics B 107 365-380

[12] Bollini C G and Giambiagi J J 1977 Nuclear Physics B 123 311-319

[13] Sokolov V V 1976 Soviet Journal of Nuclear Physics 23 330-334

[14] dos Santos R P 1982 Alguns Aspectos da Teoria Clássica do Monopolo Magnético de Dirac Master thesis IFT - Instituto de Física Teórica São Paulo URL http://www.fisica-interessante. com/dissertacao-monopolo-magnetico-dirac-classico.html

[15] Thomson J J 1909 Elements of the Mathematical Theory of Electricity and Magnetism 4th ed (Cambridge, UK: CUP - Cambridge University Press)

[16] Appel P 1909 Annaes Scientíficos da Academia Polytécnica do Porto 4 129-131

[17] Schwinger J, Milton K A, Tsai W Y, DeRaad L L and Clark D C 1976 Annals of Physics (New York) 101 451-495

[18] Sommerfeld A 1923 Atomic Structure and Spectral Lines (London: Methuen)

[19] Pereira J G 1982 Monopolo Magnético de Dirac: Algumas Aplicações do Formalismo de Wu e Yang Master thesis IFT - Instituto de Física Teórica São Paulo 